\title{
Theory of electromagnetic insertion devices and the corresponding synchrotron radiation
}

\author{
Muhammad Shumail ${ }^{*}$ and Sami G. Tantawi ${ }^{\dagger}$ \\ SLAC National Accelerator Laboratory, Menlo Park, California 94025, USA
}

(Received 15 April 2016; published 27 July 2016)

\begin{abstract}
Permanent magnet insertion devices (IDs), which are the main radiation generating devices in synchrotron light sources and free-electron lasers, use a time-invariant but space-periodic magnetic field to wiggle relativistic electrons for short-wavelength radiation generation. Recently, a high power microwave based undulator has also been successfully demonstrated at SLAC which promises the advantage of dynamic tunability of radiation spectrum and polarization. Such IDs employ transverse elecromagnetic fields which are periodic in both space and time to undulate the electrons. In this paper we develop a detailed theory of the principle of electromagnetic IDs from first principles for both linear and circular polarization modes. The electromagnetic equivalent definitions of undulator period $\left(\lambda_{u}\right)$ and undulator deflection parameter $(K)$ are derived. In the inertial frame where the average momentum of the electron is zero, we obtain the figure-8-like trajectory for the linear polarization mode and the circular trajectory for the circular polarization mode. The corresponding radiation spectra and the intensity of harmonics is also calculated.
\end{abstract}

DOI: 10.1103/PhysRevAccelBeams.19.074001

\section{INTRODUCTION}

The conventional insertion devices (IDs) (wigglers or undulators) consist of a series of alternating magnetic poles that induce a periodic local deflection in a relativistic electron beam of a synchrotron to generate intense radiation in a specific range of the spectrum. The IDs are characterized as either wigglers or undulators with no fundamental difference between the two. In undulators, the deflection is weak and the radiation interference is more pronounced as compared to the wigglers [1]. The undulators were first proposed in 1951 [2] and then demonstrated in 1953 [3] by Motz. The undulators can also be used after a linear accelerator (linac), to generate a coherent free-electron laser radiation, as first demonstrated by Madey in 1977. Today, the technology of permanent magnet undulators (PMUs) has become an established art and is being pushed to its limits to satisfy the requirements of the fourth generation light sources. The prime examples of such fourth generation light sources are the Linac Coherent Light Source (LCLS) [4] and the SPring-8 Angstrom Compact Free-Electron Laser (SACLA) [5]. Despite the great success of these modern X-ray sources, the intrinsic nature of the magnetostatic fields poses many

\footnotetext{
*Present address: Habib University, Karachi. shumail@alumni.stanford.edu;

tantawi@slac.stanford.edu

Published by the American Physical Society under the terms of the Creative Commons Attribution 3.0 License. Further distribution of this work must maintain attribution to the author(s) and the published article's title, journal citation, and DOI.
}

limitations. Permanent magnets do not allow the much sought after feature of shorter undulator period, which allows for the economical lower energy systems, while maintaining adequate aperture for the electron beam and without compromising on the field strength [6]. Also, the dynamic control is slow and limited with PMUs. Fast dynamic control of the radiation can offer exciting scientific opportunities. These limitations could be overcome by the use of high-power guided microwaves to produce a periodical transverse wiggling field. Stimulated emission by a microwave cavity from a relativistic beam was originally suggested in 1968 [7]. But due to the challenges associated with the confinement and control of high-power microwaves, the implementation of a state-of-the-art microwave based insertion device has remained elusive for a long time. Recently, however, we have demonstrated a practical, tunable microwave-based undulator with undulator period $\lambda_{u}=13.9 \mathrm{~mm}$ and undulator deflection parameter $K=0.7$ [8]. With the revival of this interest in the implementation of tunable electromagnetic IDs, we are presenting a thorough investigation of the specific principle of such devices to serve as a theoretical reference and benchmark for similar devices.

The conventional magnetic undulators rely on the transverse magnetic fields, alternating in space but static in time, to undulate or wiggle the charged particles, usually electrons, which are generally moving at relativistic speeds. If the primary direction of motion of the electrons is taken to be along the $z$-axis then a magnetic field directed along the $y$-axis but alternating as a function of the $z$-axis would cause the electrons to wiggle along the $x$-axis. This leads to a narrow beam of radiation along the forward direction of 
the electrons [9]. Depending upon the relativistic speed of the electrons, the wavelength of this radiation is orders of magnitude smaller than the period of the magnetic field alternation along the $z$-axis $[9,10]$.

The charged particles can also be wiggled by time harmonic electromagnetic waves. In this case, both the electric and magnetic fields would contribute to wiggle the electrons. Another feature of this scheme would be that the fields not only alternate in space but time as well. A device employing electromagnetic waves to undulate the electrons would be called an electromagnetic undulator. The electromagnetic fields in the frequency range of the radio frequency (rf) or microwave bands of the spectrum are more likely to be used for this purpose as the technology of generating and manipulating the electromagnetic fields is more mature in this range of the spectrum. Hence, instead of electromagnetic undulators, we generally use the term rf or microwave undulators.

The principle of microwave undulators to produce synchrotron radiation can be best understood in the inertial frame where average electron velocity is zero. In this frame, the electron causes Thomson scattering of the Dopplershifted microwaves. The scattered radiation, Dopplershifted back to the lab frame is the highly directional and very high frequency synchrotron radiation.

In this paper, we explain the fundamental principles of a microwave undulator and derive the equivalent mathematical definitions of various parameters, like $\lambda_{u}$ and $K$, that are also used to characterize the conventional magnetic undulators. Earlier, Batchelor has derived the equivalent equations of $\lambda_{u}$ and $K$ for linear polarized TE modes in rectangular waveguides [11]. Pellegrini has also done a similar analysis for the particular case of circular polarized fundamental TE mode in a square waveguide [12]. However, our analysis is more generic and we take these equations further to obtain the trajectory of an electron for both linear and circular polarization. Moreover, we have also investigated the spectrum of the synchrotron radiation and the far-field intensity of harmonics versus the observation angle. For both linear and circular polarization balanced hybrid $\mathrm{HE}_{1 n}$ modes, the electron trajectory in a microwave undulator and hence the corresponding radiation characteristics turn out to be similar to those of PMUs.

\section{ELECTROMAGNETIC FIELDS ON AXIS}

It has been discussed that the dipole modes, like $\mathrm{TM}_{1 n}$, $\mathrm{TE}_{1 n}$, and $\mathrm{HE}_{1 n}$, are the most simple and interesting modes for microwave undulator application [13]. For these modes the fields, near the vicinity of the axis ( $z$-axis) of the device along which the electrons are supposed to travel, are transverse and their expressions are given in the following equations, where $\omega$ is the angular $\mathrm{rf}$ frequency, $\beta$ is the propagation constant, $t$ is time, and $\boldsymbol{E}_{\text {axis }}$ and $\boldsymbol{B}_{\text {axis }}$ are the electric and magnetic fields, respectively, on or near the axis.
Linear polarization.-

$$
\begin{aligned}
\boldsymbol{E}_{\text {axis }}(z, t) & =-E_{\perp} \sin (\beta z) \cos (\omega t) \hat{\boldsymbol{x}} \\
& =E_{\perp} \frac{\sin (\omega t-\beta z)-\sin (\omega t+\beta z)}{2} \hat{\boldsymbol{x}} \\
\boldsymbol{B}_{\text {axis }}(z, t) & =B_{\perp} \cos (\beta z) \sin (\omega t) \hat{\boldsymbol{y}} \\
& =B_{\perp} \frac{\sin (\omega t-\beta z)+\sin (\omega t+\beta z)}{2} \hat{\boldsymbol{y}} .
\end{aligned}
$$

Circular polarization.-

$$
\begin{aligned}
\boldsymbol{E}_{\text {axis }}(z, t)= & -E_{\perp} \sin (\beta z)[\cos (\omega t) \hat{\boldsymbol{x}}+\sin (\omega t) \hat{\boldsymbol{y}}] \\
= & E_{\perp} \frac{\sin (\omega t-\beta z)-\sin (\omega t+\beta z)}{2} \hat{\boldsymbol{x}} \\
& -E_{\perp} \frac{\cos (\omega t-\beta z)-\cos (\omega t+\beta z)}{2} \hat{\boldsymbol{y}} \\
\boldsymbol{B}_{\text {axis }}(z, t)= & B_{\perp} \cos (\beta z)[\cos (\omega t) \hat{\boldsymbol{x}}+\sin (\omega t) \hat{\boldsymbol{y}}] \\
= & B_{\perp} \frac{\sin (\omega t-\beta z)+\sin (\omega t+\beta z)}{2} \hat{\boldsymbol{y}} \\
& +B_{\perp} \frac{\cos (\omega t-\beta z)+\cos (\omega t+\beta z)}{2} \hat{\boldsymbol{x}} .
\end{aligned}
$$

It is evident that $E_{\perp}$ and $B_{\perp}$ are the peak standing wave amplitudes of the respective fields. We will also use the symbols for the free space rf wavelength $(\lambda)$ and the wave number $(k \equiv 2 \pi / \lambda)$.

We are interested to explore the motion of an electron under the influence of these fields which have been described in the lab inertial frame. The electron moves at a relativistic speed along the positive $z$-axis. It turns out that there is another inertial frame, defined as electron frame, in which its motion is a stable closed orbit. Our strategy is to first solve the equations of motion for the electron in the electron frame. We choose the axes of the electron frame to coincide with those of the lab frame at time $t=0$. Moreover, the electron frame is assumed to be moving with a velocity $v_{0} \hat{z}$ with respect to the lab frame. Thus, as per our definition of the electron frame where the electron moves in a closed orbit, $v_{0} \hat{z}$ is also the average velocity of electron in the lab frame. Since the electron is moving at a relativistic speed, therefore $v_{0}$ is very close to $c$, the speed of light in vacuum. To represent a quantity in the electron frame, we will add a prime $(')$ to the corresponding symbol of that quantity in the lab frame.

Lorentz transformations [14] yield the following expressions for the fields in the electron frame:

$$
\begin{aligned}
& z=\gamma_{0}\left(z^{\prime}+v_{0} t^{\prime}\right) \\
& t=\gamma_{0}\left(t^{\prime}+z^{\prime} v_{0} / c^{2}\right)
\end{aligned}
$$




$$
\begin{aligned}
& \boldsymbol{E}_{\text {axis }}^{\prime}\left(z^{\prime}, t^{\prime}\right)=\gamma_{0}\left\{\boldsymbol{E}_{\text {axis }}(z, t)+c \hat{z} \times \boldsymbol{B}_{\text {axis }}(z, t)\right\} \\
& \boldsymbol{B}_{\text {axis }}^{\prime}\left(z^{\prime}, t^{\prime}\right)=\gamma_{0}\left\{\boldsymbol{B}_{\text {axis }}(z, t)-\frac{\hat{z}}{c} \times \boldsymbol{E}_{\text {axis }}(z, t)\right\},
\end{aligned}
$$

where $\gamma_{0} \equiv 1 / \sqrt{1-v_{0}^{2} / c^{2}}$.

\section{LINEAR POLARIZATION}

Using $v_{0} \approx c$ and Eq. (1), Eq. (3) can be written as

$$
\begin{aligned}
\boldsymbol{E}_{\text {axis }}^{\prime}\left(z^{\prime}, t^{\prime}\right)= & -c \gamma_{0} B_{u}\left[\sin \left\{\omega_{\text {rad }}^{\prime}\left(t^{\prime}+z^{\prime} / c\right)\right\}\right. \\
& \left.+\delta \sin \left\{\epsilon \omega_{\mathrm{rad}}^{\prime}\left(t^{\prime}+z^{\prime} / c\right)\right\}\right] \hat{\boldsymbol{x}} \\
\boldsymbol{B}_{\text {axis }}^{\prime}\left(z^{\prime}, t^{\prime}\right)= & \gamma_{0} B_{u}\left[\sin \left\{\omega_{\mathrm{rad}}^{\prime}\left(t^{\prime}+z^{\prime} / c\right)\right\}\right. \\
& \left.+\delta \sin \left\{\epsilon \omega_{\mathrm{rad}}^{\prime}\left(t^{\prime}+z^{\prime} / c\right)\right\}\right] \hat{\boldsymbol{y}},
\end{aligned}
$$

where

$$
\begin{aligned}
B_{u} & \equiv \frac{B_{\perp}+E_{\perp} / c}{2} \\
\omega_{\mathrm{rad}}^{\prime} & \equiv \frac{2 \pi c \gamma_{0}}{\lambda_{u}} \\
\lambda_{u} & \equiv \frac{\lambda}{1+\beta / k} \\
\epsilon & \equiv \frac{1-\beta / k}{1+\beta / k} \\
\delta & \equiv \frac{B_{\perp}-E_{\perp} / c}{B_{\perp}+E_{\perp} / c}= \begin{cases}\epsilon & \mathrm{TM}_{1 n} \\
-\epsilon & \mathrm{TE}_{1 n} \\
\frac{1-\Lambda}{1+\Lambda} \epsilon & \mathrm{HE}_{1 n}\end{cases}
\end{aligned}
$$

and $\Lambda$ is the mode hybrid factor.

In Eq. (4), the terms proportional to $\delta$ are due to the forward traveling wave, while the other ones are due to the backward traveling wave. When only backward traveling wave is present or when $\delta \ll 1$, which is usually the case, the orbital angular frequency of the electron, in the electron frame, would be $\omega_{\text {rad }}^{\prime}$ as this is the angular frequency of the driving electromagnetic field. Thus, the electron yields a radiation with angular frequency $\omega_{\text {rad }}^{\prime}$ in the electron frame. To obtain the angular frequency of this radiation in the lab frame, as observed at an angle $\theta \ll 1$ from the axis, we employ Eq. (A3):

$$
\omega_{\mathrm{rad}}=\omega_{\mathrm{rad}}^{\prime} \frac{2 \gamma_{0}}{1+\left(\gamma_{0} \theta\right)^{2}} .
$$

The corresponding wavelength of the radiation in the lab frame is given as

$$
\lambda_{\mathrm{rad}} \equiv \frac{2 \pi c}{\omega_{\mathrm{rad}}}=\frac{\lambda_{u}}{2 \gamma_{0}^{2}}\left[1+\left(\gamma_{0} \theta\right)^{2}\right]
$$

Using $-e$ and $m$ for the charge and mass of the electron, respectively, the equations of motion for the electron in the electron frame can be written in terms of the rate of change of momentum $\left(\boldsymbol{p}^{\prime}\right)$ and energy $\left(T^{\prime}\right)$ as [15]

$$
\begin{aligned}
& \frac{d \boldsymbol{p}^{\prime}}{d t^{\prime}}=-e\left(\boldsymbol{E}_{a x i s}^{\prime}+\boldsymbol{v}^{\prime} \times \boldsymbol{B}_{a x i s}^{\prime}\right) \\
& \frac{d T^{\prime}}{d t^{\prime}}=-e \boldsymbol{v}^{\prime} \cdot \boldsymbol{E}_{a x i s}^{\prime} .
\end{aligned}
$$

Here, $\boldsymbol{v}^{\prime}$ is the velocity of the electron along the closed orbit in the electron frame. Since there is no force along the $y$-axis, the motion of the electron will remain confined in the $z^{\prime}-x^{\prime}$ plane if it does not have any initial velocity component along the $y$-axis. Also note that though Eq. (8b) is not an independent equation and can be obtained from Eq. (8a), it will help us simplify the analysis.

Now we substitute Eq. (4) in Eq. (8):

$$
\begin{aligned}
\frac{d \gamma^{\prime} m v_{x}^{\prime}}{d t^{\prime}}= & e \gamma_{0} c\left(1+\frac{v_{z}^{\prime}}{c}\right) B_{u}\left[\sin \left\{\omega_{\mathrm{rad}}^{\prime}\left(t^{\prime}+\frac{z^{\prime}}{c}\right)\right\}\right. \\
& \left.+\delta \sin \left\{\epsilon \omega_{\mathrm{rad}}^{\prime}\left(t^{\prime}+\frac{z^{\prime}}{c}\right)\right\}\right] \\
\frac{d \gamma^{\prime} m v_{z}^{\prime}}{d t^{\prime}}= & -e \gamma_{0} v_{x}^{\prime} B_{u}\left[\sin \left\{\omega_{\mathrm{rad}}^{\prime}\left(t^{\prime}+\frac{z^{\prime}}{c}\right)\right\}\right. \\
& \left.+\delta \sin \left\{\epsilon \omega_{\mathrm{rad}}^{\prime}\left(t^{\prime}+\frac{z^{\prime}}{c}\right)\right\}\right] \\
\frac{d \gamma^{\prime} m c^{2}}{d t^{\prime}}= & e \gamma_{0} c v_{x}^{\prime} B_{u}\left[\sin \left\{\omega_{\mathrm{rad}}^{\prime}\left(t^{\prime}+\frac{z^{\prime}}{c}\right)\right\}\right. \\
& \left.+\delta \sin \left\{\epsilon \omega_{\mathrm{rad}}^{\prime}\left(t^{\prime}+\frac{z^{\prime}}{c}\right)\right\}\right],
\end{aligned}
$$

where

$$
\gamma^{\prime} \equiv \frac{1}{\sqrt{1-\frac{v_{x}^{\prime 2}+v_{z}^{\prime 2}}{c^{2}}}} .
$$

Multiplying Eq. (9b) by $c$ and adding to Eq. (9c) yields a constant of motion:

$$
A=\gamma^{\prime}\left(1+\frac{v_{z}^{\prime}}{c}\right)>1
$$

Corresponding to the velocity, $v$, of the electron in the lab frame we define $\gamma \equiv \frac{1}{\sqrt{1-\frac{v 2}{c^{2}}}}$. Note that $\gamma$ is the energy of the electron measured in units of electron mass 
$\left(m c^{2} \approx 0.511 \mathrm{MeV}\right)$. According to the Lorentz transformations, the energy in the lab frame is given in terms of the energy and momentum in the electron frame as follows:

$\gamma m c^{2}=\gamma_{0}\left(\gamma^{\prime} m c^{2}+v_{0} \gamma^{\prime} m v_{z}^{\prime}\right)=\gamma_{0} \gamma^{\prime} m c^{2}\left(1+\frac{v_{0}}{c} \frac{v_{z}}{c}\right)$.

Using $v_{0} \approx c$ and Eq. (11), we get

$$
\gamma_{0}=\gamma / A .
$$

Substituting Eq. (13) in Eq. (7) yields

$$
\lambda_{\mathrm{rad}}=\frac{\lambda_{u}}{2 \gamma^{2}}\left[A^{2}+(\gamma \theta)^{2}\right] .
$$

Before solving the equations of motion, let us define some unitless quantities for the sake of notational ease:

$$
\begin{aligned}
& u_{x}^{\prime} \equiv v_{x}^{\prime} / c \\
& u_{z}^{\prime} \equiv v_{z}^{\prime} / c \\
& \tau^{\prime} \equiv \omega_{\mathrm{rad}}^{\prime} t^{\prime}=\frac{2 \pi c \gamma}{A \lambda_{u}} t^{\prime} \\
& \xi^{\prime} \equiv \frac{\omega_{\mathrm{rad}}^{\prime}}{c} x^{\prime}=\frac{2 \pi \gamma}{A \lambda_{u}} x^{\prime}=\xi^{\prime}(0)+\int_{0}^{\tau^{\prime}} u_{x}^{\prime}\left(\tau^{\prime \prime}\right) d \tau^{\prime \prime} \\
& \eta^{\prime} \equiv \frac{\omega_{\mathrm{rad}}^{\prime}}{c} z^{\prime}=\frac{2 \pi \gamma}{A \lambda_{u}} z^{\prime}=\eta^{\prime}(0)+\int_{0}^{\tau^{\prime}} u_{z}^{\prime}\left(\tau^{\prime \prime}\right) d \tau^{\prime \prime} \\
& K \equiv \frac{e}{2 \pi m c} \lambda_{u} B_{u}=0.934 \lambda_{u}(\mathrm{~cm}) B_{u}(\mathrm{~T}) .
\end{aligned}
$$

The normalized spatial coordinates $\left(\xi^{\prime}\right.$ and $\left.\eta^{\prime}\right)$ can be obtained by integrating the corresponding normalized velocities $\left(u_{x}^{\prime}\right.$ and $\left.u_{z}^{\prime}\right)$ with respect to the normalized time variable $\tau^{\prime}$. The dimensionless parameter $K$ is the undulator deflection parameter defined in terms of the equivalent undulator period $\lambda_{u}$ and the equivalent magnetic field $B_{u}$. Now, using Eq. (15) we obtain the normalized version of Eqs. (9a), (9b), and (11) as follows:

$$
\begin{aligned}
\frac{d \gamma^{\prime} u_{x}^{\prime}}{d \tau^{\prime}} & =K\left(1+u_{z}^{\prime}\right)\left[\sin \left(\tau^{\prime}+\eta^{\prime}\right)+\delta \sin \left\{\epsilon\left(\tau^{\prime}+\eta^{\prime}\right)\right\}\right] \\
\frac{d \gamma^{\prime} u_{z}^{\prime}}{d \tau^{\prime}} & =-K u_{x}^{\prime}\left[\sin \left(\tau^{\prime}+\eta^{\prime}\right)+\delta \sin \left\{\epsilon\left(\tau^{\prime}+\eta^{\prime}\right)\right\}\right] \\
A & =\gamma^{\prime}\left(1+u_{z}^{\prime}\right)>1 .
\end{aligned}
$$

Equation (16a) can exactly be integrated to yield

$$
\gamma^{\prime} u_{x}^{\prime}=-K\left[\cos \left(\tau^{\prime}+\eta^{\prime}\right)+\frac{\delta}{\epsilon} \cos \left\{\epsilon\left(\tau^{\prime}+\eta^{\prime}\right)\right\}\right] .
$$

The integration constant was chosen to be zero, as by definition the average momentum vanishes in the electron frame. We can use Eqs. (10) and (16c) to express the normalized velocities $\left(u_{z}^{\prime}\right.$ and $\left.u_{x}^{\prime}\right)$ in terms of the normalized energy $\left(\gamma^{\prime}\right)$ :

$$
\begin{aligned}
& u_{z}^{\prime}=\frac{A}{\gamma^{\prime}}-1 \\
& u_{x}^{\prime}= \pm \frac{\sqrt{2 A \gamma^{\prime}-\left(A^{2}+1\right)}}{\gamma^{\prime}} .
\end{aligned}
$$

Equations (17) and (18b) imply

$$
\begin{aligned}
\gamma^{\prime}= & \frac{A^{2}+1+\frac{K^{2}}{2}\left[1+\left(\frac{\delta}{\epsilon}\right)^{2}\right]}{2 A} \\
& +\frac{K^{2}}{4 A}\left[\cos \left\{2\left(\tau^{\prime}+\eta^{\prime}\right)\right\}+4 \frac{\delta}{\epsilon} \cos \left(\tau^{\prime}+\eta^{\prime}\right) \cos \left\{\epsilon\left(\tau^{\prime}+\eta^{\prime}\right)\right\}\right. \\
& \left.+\left(\frac{\delta}{\epsilon}\right)^{2} \cos \left\{2 \epsilon\left(\tau^{\prime}+\eta^{\prime}\right)\right\}\right]
\end{aligned}
$$

Now, consider the longitudinal momentum that can be obtained from Eqs. (18a) and (19):

$$
\begin{aligned}
\gamma^{\prime} u_{z}^{\prime}= & \frac{A^{2}-\left[1+\frac{K^{2}}{2}\left\{1+\left(\frac{\delta}{\epsilon}\right)^{2}\right\}\right]}{2 A} \\
& -\frac{K^{2}}{4 A}\left[\cos \left\{2\left(\tau^{\prime}+\eta^{\prime}\right)\right\}+4 \underset{\epsilon}{\delta} \cos \left(\tau^{\prime}+\eta^{\prime}\right) \cos \left\{\epsilon\left(\tau^{\prime}+\eta^{\prime}\right)\right\}\right. \\
& \left.+\left(\frac{\delta}{\epsilon}\right)^{2} \cos \left\{2 \epsilon\left(\tau^{\prime}+\eta^{\prime}\right)\right\}\right]
\end{aligned}
$$

Since the average longitudinal momentum should be zero in the electron frame, we get the following useful result from Eq. (20):

$$
A^{2}=1+\frac{K^{2}}{2}\left[1+\left(\frac{\delta}{\epsilon}\right)^{2}\right]
$$

For the balanced hybrid $\mathrm{HE}_{1 n}$ modes, $\Lambda \approx 1 \Rightarrow \frac{\delta}{\epsilon}=$ $\frac{1-\Lambda}{1+\Lambda} \approx 0 \Rightarrow A^{2}=1+\frac{K^{2}}{2}$. Hence, Eq. (14) becomes

$$
\lambda_{\mathrm{rad}}=\frac{\lambda_{u}}{2 \gamma^{2}}\left[1+\frac{K^{2}}{2}+(\gamma \theta)^{2}\right] .
$$

Note that Eqs. (15f) and (22) are analogous to the corresponding equations for PMUs [16]. Hence, we call $B_{u}$ and $\lambda_{u}$ in Eq. (15f) the equivalent magnetic field and the equivalent undulator period, respectively. 
Now, with the assumption of the balanced hybrid $\mathrm{HE}_{1 n}$ modes $\left(\frac{\delta}{\varepsilon}=0\right)$, we get the following equations of motion which are the same as that for the PMUs:

$$
\begin{aligned}
& \gamma^{\prime}=\frac{4+2 K^{2}+K^{2} \cos \left\{2\left(\tau^{\prime}+\eta^{\prime}\right)\right\}}{4 \sqrt{1+\frac{K^{2}}{2}}} \\
& u_{x}^{\prime}=\frac{4 K \sqrt{1+\frac{K^{2}}{2}} \cos \left(\tau^{\prime}+\eta^{\prime}\right)}{4+2 K^{2}+K^{2} \cos \left\{2\left(\tau^{\prime}+\eta^{\prime}\right)\right\}} \\
& u_{z}^{\prime}=\frac{-K^{2} \cos \left\{2\left(\tau^{\prime}+\eta^{\prime}\right)\right\}}{4+2 K^{2}+K^{2} \cos \left\{2\left(\tau^{\prime}+\eta^{\prime}\right)\right\}} \\
& \xi^{\prime}=\int_{0}^{\tau^{\prime}} u_{x}^{\prime}\left(\tau^{\prime \prime}\right) d \tau^{\prime \prime} \\
& \eta^{\prime}=\int_{0}^{\tau^{\prime}} u_{z}^{\prime}\left(\tau^{\prime \prime}\right) d \tau^{\prime \prime} .
\end{aligned}
$$

Here, we have chosen $\xi^{\prime}(0)=\eta^{\prime}(0)=0$. It is evident from Eq. (23) that the motion parameters in the electron frame are a periodic function of $\tau^{\prime}$, where $u_{z}^{\prime}\left(\tau^{\prime}\right), \eta^{\prime}\left(\tau^{\prime}\right)$, and $\gamma^{\prime}\left(\tau^{\prime}\right)$ have a period of $\pi$ while $u_{x}^{\prime}\left(\tau^{\prime}\right)$ and $\xi^{\prime}\left(\tau^{\prime}\right)$ exhibit a period of $2 \pi$. It turns out that for $K \leq 1$, it is a good approximation to replace $\eta^{\prime}$, when it appears in the argument of cosine function in Eq. (23), by its fundamental harmonic only:

$$
\eta^{\prime} \approx D_{2} \sin \left(2 \tau^{\prime}\right)
$$

where, using an iterative procedure, we get the following empirical equation for the constant $D_{2}$ :

$$
D_{2}=-0.1385 K^{2}+0.0546 K^{3} .
$$

Thus for $K \leq 1$, we need to solve the following integrals, which can easily be performed numerically, to get the normalized trajectory of the electron in the electron frame:

$\xi^{\prime}\left(\tau^{\prime}\right)=\int_{0}^{\tau^{\prime}} \frac{4 K \sqrt{1+\frac{K^{2}}{2}} \cos \left[\tau^{\prime \prime}+D_{2} \sin \left(2 \tau^{\prime \prime}\right)\right]}{4+2 K^{2}+K^{2} \cos \left[2\left\{\tau^{\prime \prime}+D_{2} \sin \left(2 \tau^{\prime \prime}\right)\right\}\right]} d \tau^{\prime \prime}$

$\eta^{\prime}\left(\tau^{\prime}\right)=\int_{0}^{\tau^{\prime}} \frac{-K^{2} \cos \left[2\left\{\tau^{\prime \prime}+D_{2} \sin \left(2 \tau^{\prime \prime}\right)\right\}\right]}{4+2 K^{2}+K^{2} \cos \left[2\left\{\tau^{\prime \prime}+D_{2} \sin \left(2 \tau^{\prime \prime}\right)\right\}\right]} d \tau^{\prime \prime}$.

It turns out that for $K \leq 1$, we can replace the more precise Eqs. (23b), (23c), and (26) with the following approximate equations which are more intuitive:

$$
\begin{aligned}
& u_{x}^{\prime}\left(\tau^{\prime}\right) \approx \frac{K}{\sqrt{1+\frac{K^{2}}{2}}} \cos \left(\tau^{\prime}\right) \\
& u_{z}^{\prime}\left(\tau^{\prime}\right) \approx-\frac{K^{2}}{2\left(2+K^{2}\right)} \cos \left(2 \tau^{\prime}\right) \\
& \xi^{\prime}\left(\tau^{\prime}\right) \approx \frac{K}{\sqrt{1+\frac{K^{2}}{2}}} \sin \left(\tau^{\prime}\right) \\
& \eta^{\prime}\left(\tau^{\prime}\right) \approx-\frac{K^{2}}{4\left(2+K^{2}\right)} \sin \left(2 \tau^{\prime}\right) .
\end{aligned}
$$

Equations (27c) and (27d) are the parametric equations of the figure-8-like Lissajous curve. In the literature related to PMUs, we usually find mention of Eqs. (27a) and (27b), which are approximate, rather than the more precise version given by Eqs. (23b) and (23c) (see, for example, [17]). It turns out that, though qualitatively both sets of equations yield similar radiation characteristics, there are huge quantitative differences for higher harmonics. In this paper, we have used the more precise version of equations to determine angular and spectral radiation characteristics. Figure 1 shows the orbits of the electron in the electron frame for $K=0.5$ (blue) and $K=1.0$ (red, dashed). Note that the horizontal coordinate is stretched 10 times in comparison to the vertical coordinate. Though these orbits were obtained using the more precise Eq. (26), we get visually overlapping curves if we use the approximate but intuitive version given by Eqs. (27c) and (27d).

Now we will employ standard Lorentz transformations to find the corresponding motion parameters in the lab

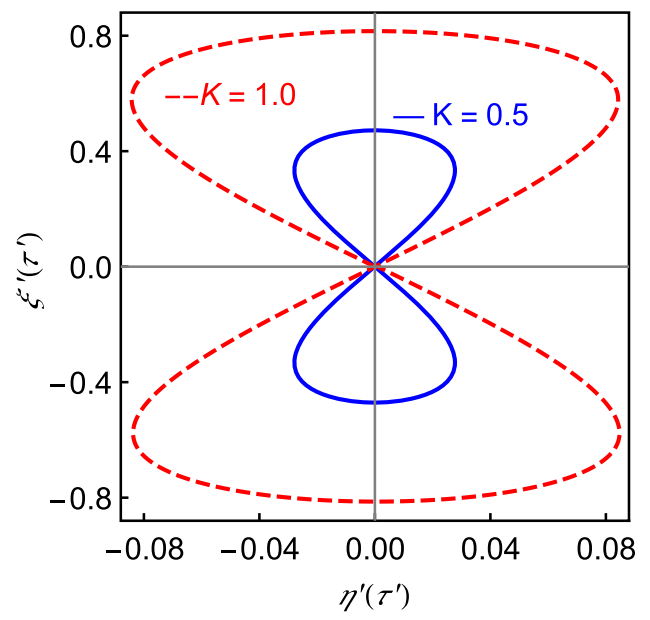

FIG. 1. Normalized orbit of the electron in the electron inertial frame for two different values of $K$. At $\tau^{\prime}=0,2 \pi, 4 \pi, \ldots$ the electron crosses the origin to enter the second quadrant from the fourth. At $\tau^{\prime}=\pi, 3 \pi, 5 \pi, \ldots$ the electron crosses the origin to enter the third quadrant from the first. 
frame. Here we will use the result derived in Eq. (13), i.e., $\gamma_{0}=\gamma / A$ and $\gamma_{0} v_{0} / c=\sqrt{\left(\frac{\gamma}{A}\right)^{2}-1}$ :

$$
\begin{aligned}
\xi & \equiv \frac{2 \pi}{\lambda_{u}} x=\frac{A}{\gamma} \xi^{\prime} \\
\zeta & \equiv \frac{2 \pi}{\lambda_{u}} y=0 \\
\eta & \equiv \frac{2 \pi}{\lambda_{u}} z=\eta^{\prime}+\tau^{\prime} \sqrt{1-\left(\frac{A}{\gamma}\right)^{2}} \\
\tau & \equiv \frac{2 \pi c}{\lambda_{u}} t=\tau^{\prime}+\eta^{\prime} \sqrt{1-\left(\frac{A}{\gamma}\right)^{2}} \\
u_{x} & \equiv \frac{v_{x}}{c}=\frac{A u_{x}^{\prime}}{\gamma\left[1+u_{z}^{\prime} \sqrt{1-\left(\frac{A}{\gamma}\right)^{2}}\right.} \\
u_{z} & \equiv \frac{v_{z}}{c}=\frac{u_{z}^{\prime}+\sqrt{1-\left(\frac{A}{\gamma}\right)^{2}}}{1+u_{z}^{\prime} \sqrt{1-\left(\frac{A}{\gamma}\right)^{2}}} \\
\alpha_{x} & \equiv \frac{\lambda_{u}}{2 \pi c^{2}} \frac{d v_{x}}{d t}=\frac{d u_{x}}{d \tau}=\frac{1}{1+u_{z}^{\prime} \sqrt{1-\left(\frac{A}{\gamma}\right)^{2}}} \frac{d u_{x}}{d \tau^{\prime}} \\
\alpha_{z} & \equiv \frac{\lambda_{u}}{2 \pi c^{2}} \frac{d v_{z}}{d t}=\frac{d u_{z}}{d \tau}=\frac{1}{1+u_{z}^{\prime} \sqrt{1-\left(\frac{A}{\gamma}\right)^{2}}} \frac{d u_{z}}{d \tau^{\prime}} .
\end{aligned}
$$

We can use Eqs. (28a) and (28c) to calculate the trajectory of the electron in the lab frame. However, using Eqs. (28a) and (27c), along with the fact that for relativistic motion $\eta \approx \tau \approx \tau^{\prime}$, we obtain following simple equation, socalled second field integral, for the trajectory of the electron in the lab frame:

$$
\xi=\frac{K}{\gamma} \sin (\eta)
$$

As expected, Eq. (29) is the same as for the case of PMUs [18]. To calculate the energy of the electron as a function of time in the lab frame, we can use Eqs. (28e) and (28f). Figure 2 shows the electron trajectory and the slight modulation in the energy of the electron in the lab frame for $K=0.5$ (blue) and $K=1.0$ (red, dashed). The period chosen corresponds to the interval $0 \leq \tau^{\prime} \leq 2 \pi$. For Fig. 2(b) the value of $\gamma$ was chosen corresponding to an electron energy of $60 \mathrm{MeV}(\gamma=117.417)$.

Having solved the trajectory, we can calculate the electric field $\left(E_{\text {rad }}\right)$ of the far-field radiation at a spacetime point $\left(x_{0}, y_{0}, z_{0}, c t_{0}\right) \equiv \frac{\lambda_{u}}{2 \pi}\left(\xi_{0}, \zeta_{0}, \eta_{0}, \tau_{0}\right)$ in the lab frame using the expression derived from LiénardWiechert potentials [19]:
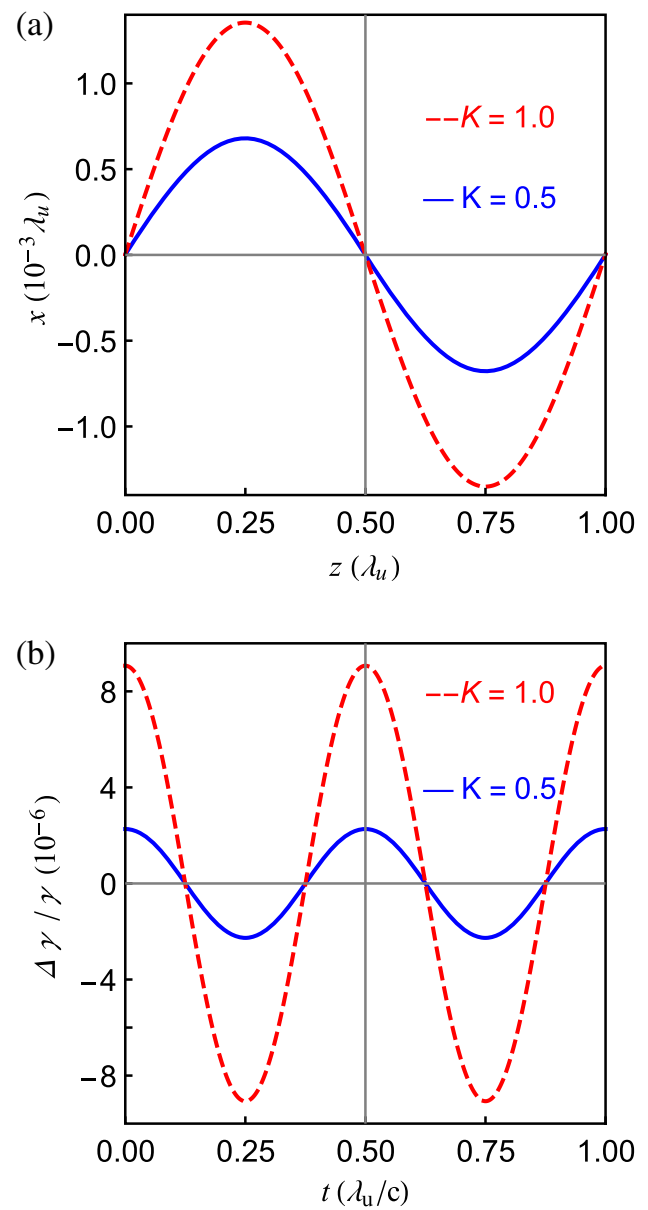

FIG. 2. Trajectory and energy modulation in the lab frame. (a) A period of electron trajectory. (b) Normalized change in the energy of the electron during a single period.

$$
\begin{aligned}
E_{\mathrm{rad}}\left(\xi_{0}, \zeta_{0}, \eta_{0}, \tau_{0}\right) & =-\frac{\pi e}{\epsilon_{0} \lambda_{u}^{2}} \frac{\boldsymbol{r} \times\{(\boldsymbol{r}-\boldsymbol{u} r) \times \boldsymbol{\alpha}\}}{(r-\boldsymbol{u} . \boldsymbol{r})^{3}} \\
\tau_{0} & =\tau+r,
\end{aligned}
$$

where

$$
\begin{aligned}
\boldsymbol{r} & \equiv\left(\xi_{0}-\xi\right) \hat{\boldsymbol{x}}+\left(\zeta_{0}-\zeta\right) \hat{\boldsymbol{y}}+\left(\eta_{0}-\eta\right) \hat{z} \\
r & \equiv|\boldsymbol{r}| \\
\boldsymbol{u} & \equiv u_{x} \hat{\boldsymbol{x}}+u_{y} \hat{\boldsymbol{y}}+u_{z} \hat{z} \\
\boldsymbol{\alpha} & \equiv \alpha_{x} \hat{\boldsymbol{x}}+\alpha_{y} \hat{\boldsymbol{y}}+\alpha_{z} \hat{z} .
\end{aligned}
$$

Here, $\epsilon_{0}$ is the free space permittivity. Note that for the current case (linear polarization) $\zeta=u_{y}=\alpha_{y}=0$.

These equations can be used to calculate, for example, the angular and spectral distribution of intensity of various harmonics at any far-field point due to a single electron. 

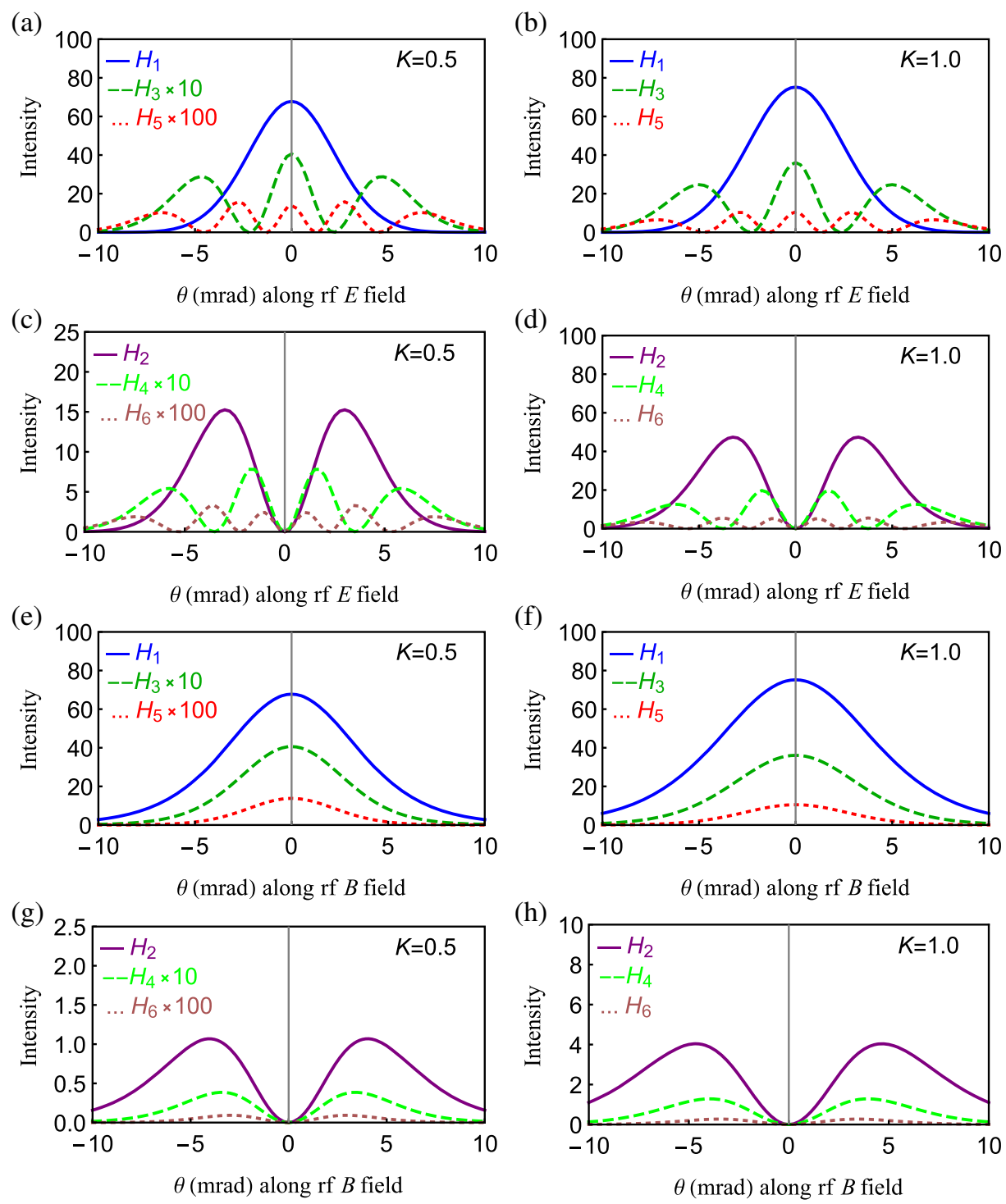

FIG. 3. Linear polarization: For a single electron the intensity (in units of $10^{-12} \mathrm{~W} \mathrm{~m}^{2} / \lambda_{u}^{4}$ ) at a distance of $100 \lambda_{u}$ of the first three odd and even harmonics vs the off-axis angle $\theta$ in a far-field transverse plane. The electron energy is $60 \mathrm{MeV}$.

Figure 3 shows the intensity of various harmonics $\left(H_{n}\right)$ versus the off-axis angle $\theta$ in a far-field transverse plane, $100 \lambda_{u}$ ahead of the electron. The electron energy is chosen to be $60 \mathrm{MeV}$ (typical energy of the electron beam at NLCTA, SLAC). The subscript on $H$ represents the number of harmonic. It is evident that while odd harmonics are more intense on the axis, the even harmonics are more intense off axis. Moreover, the intensity of any even harmonic along the rf $E$ plane is an order of magnitude higher than its intensity along the $\operatorname{rf} B$ plane. Figure 4 shows the on-axis intensity of various odd harmonics against different values of $K$. Generally, the intensity of the harmonics increases as we increase $K$. At higher values of $K$, the intensity of higher harmonics increases at the expense of the intensity of the lower harmonics. Note that the intensity of the odd harmonics $H_{3}$ and $H_{5}$ increases by an order of magnitude as we go from $K=0.5$ to $K=1.0$. However, the intensity of the fundamental harmonic is maximum at $K \approx 0.75$.

We can also calculate the spectrum of the radiation as observed along a line in the far-field transverse plane by calculating the intensity and the wavelength of the fundamental harmonic along that line. According to Eq. (22) the wavelength increases as we go off axis. Figure 5 shows the spectra calculated for $K=0.5$ (blue) and $K=1.0$ (red, dashed). For these calculations, $\gamma=117.417$ (corresponding to $60 \mathrm{MeV}$ electron energy) and $\lambda_{u}=1.39 \mathrm{~cm}$ with 78 undulator periods as per our demonstrated microwave undulator [8]. The spectrum was calculated assuming the observation line to be along $x=y$ in the far-field transverse plane. It has been observed, however, that the orientation of the observation line does not much change the profile of the 


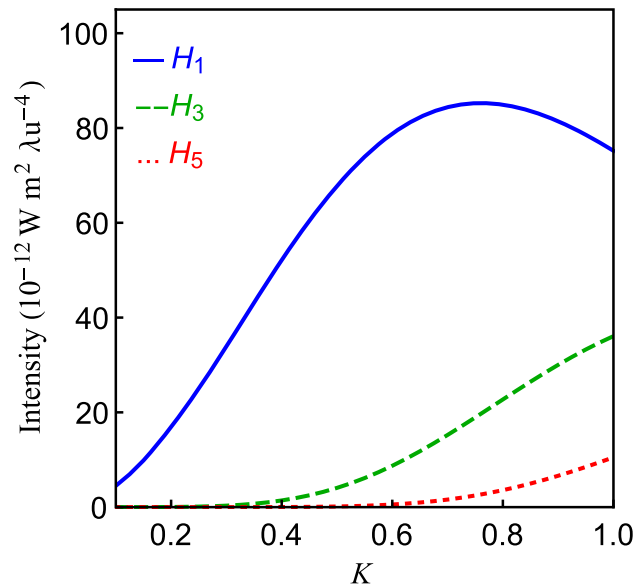

FIG. 4. Linear polarization: For a single electron the on-axis intensity of the first three odd harmonics vs $K$ at a distance of $100 \lambda_{u}$. The electron energy is $60 \mathrm{MeV}$.

spectrum. Each spectrum shown in Fig. 5 is in fact the convolution of the so-called line function spectrum, corresponding to the duration of 78 undulating cycles, with the corresponding continuous theoretical spectrum. The onaxis radiation wavelength as determined by Eq. (22) is $567 \mathrm{~nm}(756 \mathrm{~nm})$ corresponding to $K=0.5(K=1.0)$. Note that the peaks of the corresponding spectra occur a little right to these wavelengths which are marked by dashed vertical lines in Fig. 5.

\section{CIRCULAR POLARIZATION}

Using $v_{0} \approx c$ and Eq. (2), Eq. (3) can be written as

$$
\begin{aligned}
\boldsymbol{E}_{\text {axis }}^{\prime}\left(z^{\prime}, t^{\prime}\right)= & -c \gamma_{0} B_{u}\left[\left(\begin{array}{c}
\sin \left\{\omega_{\mathrm{rad}}^{\prime}\left(t^{\prime}+z^{\prime} / c\right)\right\} \hat{\boldsymbol{x}} \\
-\cos \left\{\omega_{\mathrm{rad}}^{\prime}\left(t^{\prime}+z^{\prime} / c\right)\right\} \hat{\boldsymbol{y}}
\end{array}\right)\right. \\
& \left.+\delta\left(\begin{array}{c}
\sin \left\{\epsilon \omega_{\mathrm{rad}}^{\prime}\left(t^{\prime}+z^{\prime} / c\right)\right\} \hat{\boldsymbol{x}} \\
-\cos \left\{\epsilon \omega_{\mathrm{rad}}^{\prime}\left(t^{\prime}+z^{\prime} / c\right)\right\} \hat{\boldsymbol{y}}
\end{array}\right)\right] \\
\boldsymbol{B}_{\text {axis }}^{\prime}\left(z^{\prime}, t^{\prime}\right)= & \gamma_{0} B_{u}\left[\left(\begin{array}{c}
\cos \left\{\omega_{\mathrm{rad}}^{\prime}\left(t^{\prime}+z^{\prime} / c\right)\right\} \hat{\boldsymbol{x}} \\
+\sin \left\{\omega_{\mathrm{rad}}^{\prime}\left(t^{\prime}+z^{\prime} / c\right)\right\} \hat{\boldsymbol{y}}
\end{array}\right)\right. \\
& \left.+\delta\left(\begin{array}{c}
\cos \left\{\epsilon \omega_{\mathrm{rad}}^{\prime}\left(t^{\prime}+z^{\prime} / c\right)\right\} \hat{\boldsymbol{x}} \\
+\sin \left\{\epsilon \omega_{\mathrm{rad}}^{\prime}\left(t^{\prime}+z^{\prime} / c\right)\right\} \hat{\boldsymbol{y}}
\end{array}\right)\right],
\end{aligned}
$$

where the definitions given in Eq. (5) hold. Moreover, the comments following Eq. (5) and the subsequent equations (6), (7), and (8) are also valid for the circular polarization case.

Now we substitute Eq. (32) in Eq. (8):

$$
\begin{aligned}
\frac{d \gamma^{\prime} m v_{x}^{\prime}}{d t^{\prime}}= & e \gamma_{0} c\left(1+\frac{v_{z}^{\prime}}{c}\right) B_{u}\left[\sin \left\{\omega_{\mathrm{rad}}^{\prime}\left(t^{\prime}+\frac{z^{\prime}}{c}\right)\right\}\right. \\
& \left.+\delta \sin \left\{\epsilon \omega_{\mathrm{rad}}^{\prime}\left(t^{\prime}+\frac{z^{\prime}}{c}\right)\right\}\right]
\end{aligned}
$$

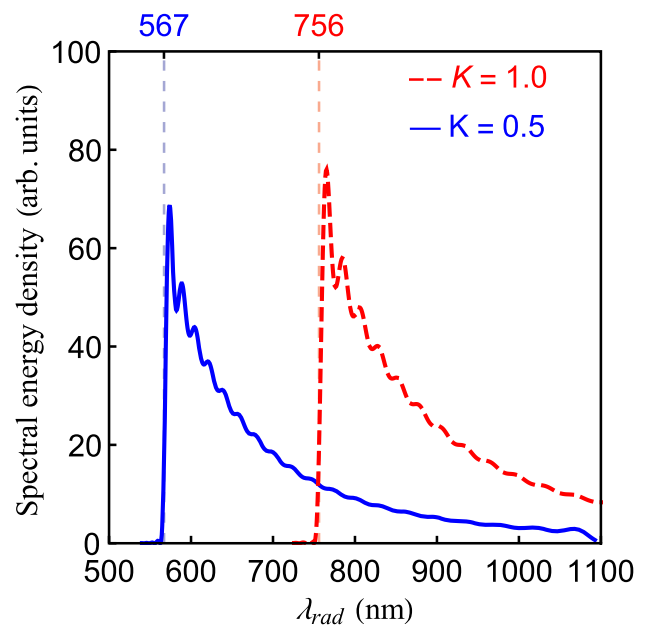

FIG. 5. Linear polarization: Far-field spectral energy density (energy/wavelength), as measured along the $x=y$ plane, for two different values of $K$. The electron energy is $60 \mathrm{MeV}$ while $\lambda_{u}=1.39 \mathrm{~cm}$.

$$
\begin{aligned}
\frac{d \gamma^{\prime} m v_{y}^{\prime}}{d t^{\prime}}= & -e \gamma_{0} c\left(1+\frac{v_{z}^{\prime}}{c}\right) B_{u}\left[\cos \left\{\omega_{\mathrm{rad}}^{\prime}\left(t^{\prime}+\frac{z^{\prime}}{c}\right)\right\}\right. \\
& \left.+\delta \cos \left\{\epsilon \omega_{\mathrm{rad}}^{\prime}\left(t^{\prime}+\frac{z^{\prime}}{c}\right)\right\}\right] \\
\frac{d \gamma^{\prime} m v_{z}^{\prime}}{d t^{\prime}}= & -e \gamma_{0} B_{u}\left[v _ { x } ^ { \prime } \left[\sin \left\{\omega_{\mathrm{rad}}^{\prime}\left(t^{\prime}+\frac{z^{\prime}}{c}\right)\right\}\right.\right. \\
& \left.+\delta \sin \left\{\epsilon \omega_{\mathrm{rad}}^{\prime}\left(t^{\prime}+\frac{z^{\prime}}{c}\right)\right\}\right] \\
& -v_{y}^{\prime}\left[\cos \left\{\omega_{\mathrm{rad}}^{\prime}\left(t^{\prime}+\frac{z^{\prime}}{c}\right)\right\}\right. \\
& \left.\left.+\delta \cos \left\{\epsilon \omega_{\mathrm{rad}}^{\prime}\left(t^{\prime}+\frac{z^{\prime}}{c}\right)\right\}\right]\right]
\end{aligned}
$$

$$
\begin{aligned}
\frac{d \gamma^{\prime} m c^{2}}{d t^{\prime}}= & e \gamma_{0} c B_{u}\left[v _ { x } ^ { \prime } \left[\sin \left\{\omega_{\text {rad }}^{\prime}\left(t^{\prime}+\frac{z^{\prime}}{c}\right)\right\}\right.\right. \\
& \left.+\delta \sin \left\{\epsilon \omega_{\text {rad }}^{\prime}\left(t^{\prime}+\frac{z^{\prime}}{c}\right)\right\}\right] \\
& -v_{y}^{\prime}\left[\cos \left\{\omega_{\text {rad }}^{\prime}\left(t^{\prime}+\frac{z^{\prime}}{c}\right)\right\}\right. \\
& \left.\left.+\delta \cos \left\{\epsilon \omega_{\text {rad }}^{\prime}\left(t^{\prime}+\frac{z^{\prime}}{c}\right)\right\}\right]\right],
\end{aligned}
$$

where

$$
\gamma^{\prime} \equiv \frac{1}{\sqrt{1-\frac{v_{x}^{\prime 2}+v_{y}^{\prime 2}+v_{z}^{\prime 2}}{c^{2}}}} .
$$

Multiplying Eq. (33c) by $c$ and adding to Eq. (33d) yields the same constant of motion as in Eq. (11). 
Equations (12), (13), and (14) also hold for the circular polarization case. Moreover, just like the linear polarization case, we will make use of the definitions given in Eq. (15) along with the following:

$$
\begin{aligned}
& u_{y}^{\prime} \equiv v_{y}^{\prime} / c \\
& \zeta^{\prime} \equiv \frac{\omega_{\mathrm{rad}}^{\prime}}{c} y^{\prime}=\frac{2 \pi \gamma}{A \lambda_{u}} y^{\prime}=\zeta^{\prime}(0)+\int_{0}^{\tau^{\prime}} u_{y}^{\prime}\left(\tau^{\prime \prime}\right) d \tau^{\prime \prime} .
\end{aligned}
$$

On one hand, using Eqs. (34) and (11), we get the following normalized equation:

$$
\gamma^{\prime 2}\left(u_{x}^{\prime 2}+u_{y}^{\prime 2}\right)=2 A \gamma^{\prime}-\left(1+A^{2}\right)
$$

On the other hand, consider the normalized version of Eqs. (33a) and (33b):

$\frac{d \gamma^{\prime} u_{x}^{\prime}}{d \tau^{\prime}}=K\left(1+u_{z}^{\prime}\right)\left[\sin \left(\tau^{\prime}+\eta^{\prime}\right)+\delta \sin \left\{\epsilon\left(\tau^{\prime}+\eta^{\prime}\right)\right\}\right]$

$\frac{d \gamma^{\prime} u_{y}^{\prime}}{d \tau^{\prime}}=-K\left(1+u_{z}^{\prime}\right)\left[\cos \left(\tau^{\prime}+\eta^{\prime}\right)+\delta \cos \left\{\epsilon\left(\tau^{\prime}+\eta^{\prime}\right)\right\}\right]$.

Equation (37) can exactly be integrated to yield

$$
\begin{aligned}
& \gamma^{\prime} u_{x}^{\prime}=-K\left[\cos \left(\tau^{\prime}+\eta^{\prime}\right)+\frac{\delta}{\epsilon} \cos \left\{\epsilon\left(\tau^{\prime}+\eta^{\prime}\right)\right\}\right] \\
& \gamma^{\prime} u_{y}^{\prime}=-K\left[\sin \left(\tau^{\prime}+\eta^{\prime}\right)+\frac{\delta}{\epsilon} \sin \left\{\epsilon\left(\tau^{\prime}+\eta^{\prime}\right)\right\}\right] .
\end{aligned}
$$

Equation (38) implies

$$
\gamma^{\prime 2}\left(u_{x}^{\prime 2}+u_{y}^{\prime 2}\right)=K^{2}\left[1+\left(\frac{\delta}{\epsilon}\right)^{2}+2 \frac{\delta}{\epsilon} \cos \left\{(1-\epsilon)\left(\tau^{\prime}+\eta^{\prime}\right)\right\}\right] .
$$

Now, comparing Eqs. (36) and (39) yields

$$
\gamma^{\prime}=\frac{\left(1+A^{2}\right)+K^{2}\left[1+\left(\frac{\delta}{\epsilon}\right)^{2}+2 \frac{\delta}{\epsilon} \cos \left\{(1-\epsilon)\left(\tau^{\prime}+\eta^{\prime}\right)\right\}\right]}{2 A} .
$$

Then consider the longitudinal momentum that can be obtained from Eqs. (40) and (11): $\gamma^{\prime} u_{z}^{\prime}=\frac{A^{2}-1-K^{2}\left[1+\left(\frac{\delta}{\epsilon}\right)^{2}+2 \frac{\delta}{\epsilon} \cos \left\{(1-\epsilon)\left(\tau^{\prime}+\eta^{\prime}\right)\right\}\right]}{2 A}$.

Since the average longitudinal momentum should be zero in the electron frame, we get the following useful result from Eq. (41):

$$
A^{2}=1+K^{2}\left[1+\left(\frac{\delta}{\epsilon}\right)^{2}\right] .
$$

For the balanced hybrid $\mathrm{HE}_{1 n}$ modes, $\Lambda \approx 1 \Rightarrow \frac{\delta}{\epsilon}=$ $\frac{1-\Lambda}{1+\Lambda} \approx 0 \Rightarrow A^{2}=1+K^{2}$. Hence, Eq. (14) becomes

$$
\lambda_{\text {rad }}=\frac{\lambda_{u}}{2 \gamma^{2}}\left[1+K^{2}+(\gamma \theta)^{2}\right]
$$

Note that Eq. (43) is analogous to the corresponding equation for the circular polarized PMUs [16]. With the assumption of $\mathrm{HE}_{1 n}$ modes, we can simplify the relevant equations of motion as follows:

$$
\begin{aligned}
& \gamma^{\prime}=A=\sqrt{1+K^{2}} \\
& u_{z}^{\prime}=0 \\
& u_{x}^{\prime}=-\frac{K}{A} \cos \tau^{\prime} \\
& u_{y}^{\prime}=-\frac{K}{A} \sin \tau^{\prime} \\
& \eta^{\prime}=0 \\
& \xi^{\prime}=\frac{2 \pi \gamma}{\lambda_{u} A} x^{\prime}=-\frac{K}{A} \sin \tau^{\prime} \\
& \zeta^{\prime}=\frac{2 \pi \gamma}{\lambda_{u} A} y^{\prime}=\frac{K}{A} \cos \tau^{\prime} .
\end{aligned}
$$

It is clear from the last two equations that the electron rotates in a circle of radius $\frac{K}{2 \pi \gamma} \lambda_{u}$ in the transverse plane of the electron frame. As the transverse coordinates are preserved under the Lorentz transformation to the lab frame, this is also the radius of the helical motion of the electron in the lab frame.

Analogous to Eq. (28), we can now obtain motion parameters in the lab frame for the circular polarization case:

$$
\begin{aligned}
& \xi \equiv \frac{2 \pi}{\lambda_{u}} x=\frac{A}{\gamma} \xi^{\prime}=-\frac{K}{\gamma} \sin \tau^{\prime} \\
& \zeta \equiv \frac{2 \pi}{\lambda_{u}} y=\frac{A}{\gamma} \zeta^{\prime}=\frac{K}{\gamma} \cos \tau^{\prime}
\end{aligned}
$$



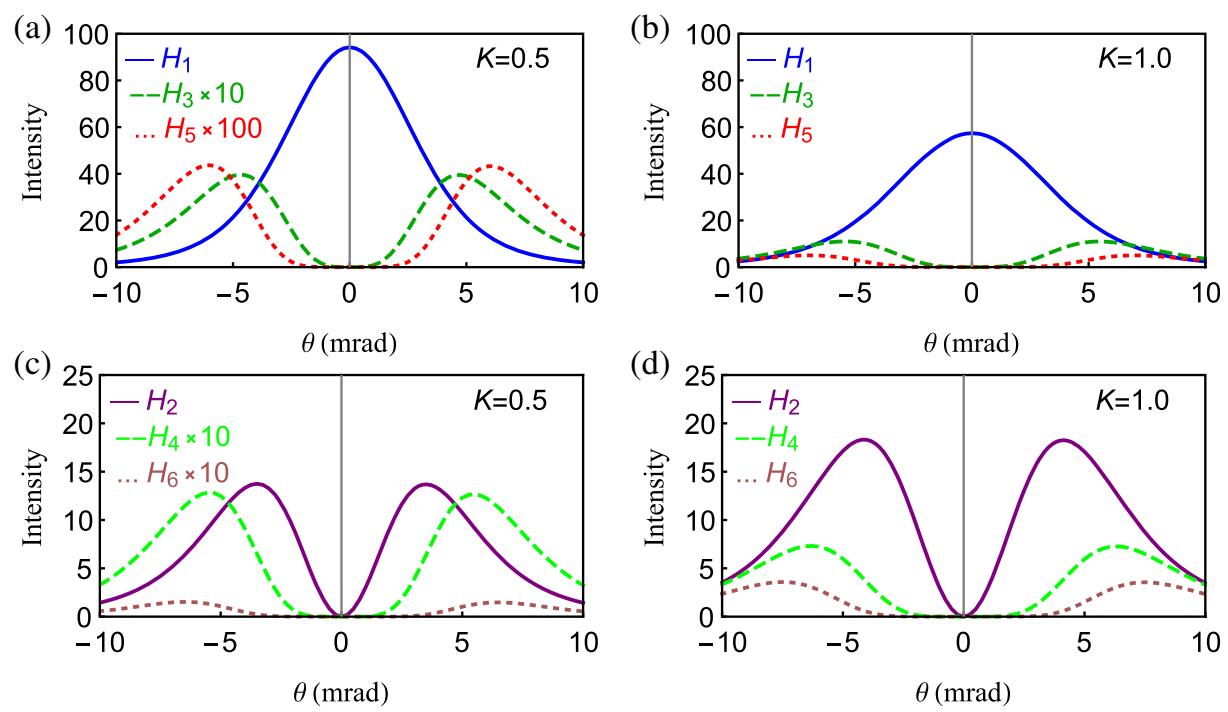

FIG. 6. Circular polarization: For a single electron the intensity (in units of $10^{-12} \mathrm{~W} \mathrm{~m}^{2} / \lambda_{u}^{4}$ ) at a distance of $100 \lambda_{u}$ of the first three odd and even harmonics versus the off-axis angle $\theta$ in a far-field transverse plane. The electron energy is $60 \mathrm{MeV}$.

$$
\begin{aligned}
& \eta \equiv \frac{2 \pi}{\lambda_{u}} z=\eta^{\prime}+\tau^{\prime} \sqrt{1-\left(\frac{A}{\gamma}\right)^{2}}=\tau^{\prime} \sqrt{1-\left(\frac{A}{\gamma}\right)^{2}} \\
& \tau \equiv \frac{2 \pi c}{\lambda_{u}} t=\tau^{\prime}+\eta^{\prime} \sqrt{1-\left(\frac{A}{\gamma}\right)^{2}}=\tau^{\prime} \\
& u_{x} \equiv \frac{v_{x}}{c}=\frac{A u_{x}^{\prime}}{\gamma\left[1+u_{z}^{\prime} \sqrt{1-\left(\frac{A}{\gamma}\right)^{2}}\right]}=-\frac{K}{\gamma} \cos \tau^{\prime} \\
& u_{y} \equiv \frac{v_{y}}{c}=\frac{A u_{y}^{\prime}}{\gamma\left[1+u_{z}^{\prime} \sqrt{1-\left(\frac{A}{\gamma}\right)^{2}}\right]}=-\frac{K}{\gamma} \sin \tau^{\prime} \\
& u_{z} \equiv \frac{v_{z}}{c}=\frac{u_{z}^{\prime}+\sqrt{1-\left(\frac{A}{\gamma}\right)^{2}}}{1+u_{z}^{\prime} \sqrt{1-\left(\frac{A}{\gamma}\right)^{2}}=\sqrt{1-\left(\frac{A}{\gamma}\right)^{2}}} \\
& \alpha_{x} \equiv \frac{\lambda_{u}}{2 \pi c^{2}} \frac{d v_{x}}{d t}=\frac{d u_{x}}{d \tau}=\frac{K}{\gamma} \sin \tau^{\prime} \\
& \alpha_{y} \equiv \frac{\lambda_{u}}{2 \pi c^{2}} \frac{d v_{y}}{d t}=\frac{d u_{y}}{d \tau}=-\frac{K}{\gamma} \cos \tau^{\prime} \\
& \alpha_{z} \equiv \frac{\lambda_{u}}{2 \pi c^{2}} \frac{d v_{z}}{d t}=\frac{d u_{z}}{d \tau}=0 .
\end{aligned}
$$

Now we can use Eqs. (30) and (31) to calculate the farfield radiation due to a single electron at a space-time point $\left(x_{0}, y_{0}, z_{0}, c t_{0}\right) \equiv \frac{\lambda_{u}}{2 \pi}\left(\xi_{0}, \zeta_{0}, \eta_{0}, \tau_{0}\right)$ in the lab frame.

In Fig. 6, we show the intensity of various harmonics versus the off-axis angle $\theta$ in a far-field transverse plane,
$100 \lambda_{u}$ ahead of the electron. The electron energy is chosen to be $60 \mathrm{MeV}$. Except for the fundamental harmonic $H_{1}$, all other harmonics are more intense off axis. As we go from $K=0.5$ to $K=1.0$, the intensity of the fundamental harmonic decreases while that of other harmonics increase by an order of magnitude. Figure 7 plots the on-axis intensity of the fundamental harmonic against different values of $K$. Note that the intensity of the fundamental harmonic reaches maximum at $K \approx 0.57$ and then decreases as we increase $K$.

As was done for the case of linear polarization, we can calculate the spectrum of the radiation along a line in the far-field transverse plane by calculating the intensity and the wavelength of the fundamental harmonic along that

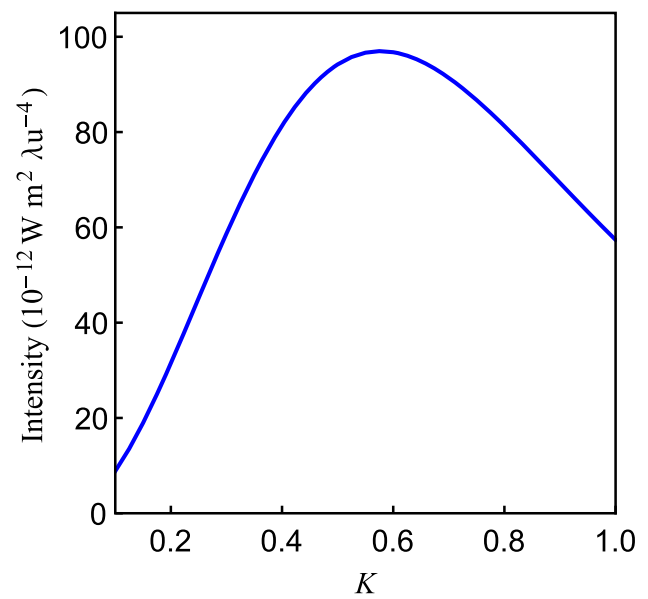

FIG. 7. Circular polarization: For a single electron the on-axis intensity of the fundamental harmonic $H_{1}$ versus $K$ at a distance of $100 \lambda_{u}$. The electron energy is $60 \mathrm{MeV}$. 


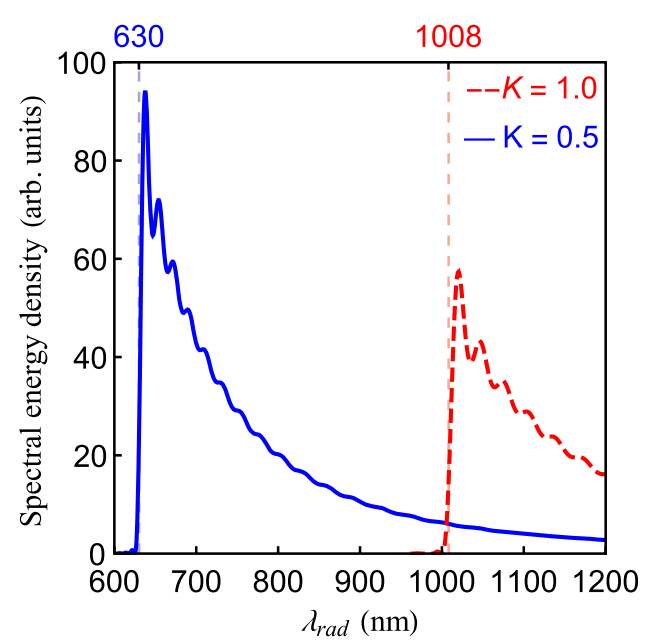

FIG. 8. Circular polarization: Far-field spectral energy density (energy/wavelength) for two different values of $K$. The electron energy is $60 \mathrm{MeV}$ while $\lambda_{u}=1.39 \mathrm{~cm}$.

line. According to Eq. (43) the wavelength increases as we go off axis. However, for the case of circular polarization, the orientation of the observation line in the far-field transverse plane does not matter. Figure 8 shows the spectra calculated for $K=0.5$ (blue) and $K=1.0$ (red, dashed). For these calculations, $\gamma=117.417$ (corresponding to $60 \mathrm{MeV}$ electron energy) and $\lambda_{u}=1.39 \mathrm{~cm}$. As in the case of linear polarization, the spectra shown in Fig. 8 are obtained by convolving the line function spectrum, corresponding to the duration of 78 cycles of undulation, with the corresponding continuous theoretical spectrum. Here again the peaks of the spectra lie a little right to the on-axis radiation wavelengths given by Eq. (43). These wavelengths are marked by vertical dashed lines in Fig. 8.

\section{CONCLUSION}

We have derived the electromagnetic equivalent definitions of undulator period $\left(\lambda_{u}\right)$ and undulator deflection parameter $(K)$ for linear and circular polarized dipole modes: $\mathrm{TM}_{1 n}, \mathrm{TE}_{1 n}$, and $\mathrm{HE}_{1 n}$. We have shown that in the electron frame, where the average momentum of the electron is zero, it traces a figure-8-like trajectory for the linear polarization mode and a circular trajectory for the circular polarization mode. The parametric equations of these trajectories are derived. For balanced hybrid $\mathrm{HE}_{1 n}$ modes, these trajectories are found similar to those in the case of PMUs. The amplitude of the sinusoidal trajectory (linear polarization) or the radius of the helical trajectory (circular polarization) is determined as $\frac{K}{2 \pi \gamma} \lambda_{u}$. We have also obtained the curves for the intensity of various harmonics versus the off-axis angle. It turns out that for the linear polarization the odd harmonics are stronger on the axis while the intensity of even harmonics is maximum at some off-axis angle. For the circular polarization only the fundamental harmonic is present on the axis. For $K>0.5$, the intensity of higher harmonics increases at the expense of that of the fundamental harmonic. The maximum intensity of the fundamental harmonic occurs for $K \approx 0.75$ and $K \approx 0.57$ for the linear and circular polarization, respectively. It is also observed that the far-field intensity of harmonics is proportional to the sixth power of electron energy. Moreover, we have presented the theoretical spectra of synchrotron radiation for various cases. In principle, one can use the equations derived in this paper to calculate the spectrum for any particular parameters of an electromagnetic insertion device. The fundamental on-axis radiation wavelength can then be estimated by correlating this theoretical spectrum with the measured one, as we have done during the data analysis of our microwave undulator [8]. It is hoped that the theoretical study presented in this paper would be a useful benchmark for the tunable IDs which are based on electromagnetic fields.

\section{ACKNOWLEDGMENTS}

The project was funded by the U.S. Department of Energy and DARPA AXiS program. M. S. is also thankful to Habib University who provided MATHEMATICA ${ }^{\circledR}$ software that was used for mathematical analysis and graph plotting in this paper.

\section{APPENDIX: DOPPLER SHIFT AT RELATIVISTIC SPEEDS AND NARROW ANGLES}

According to Lorentz transformations, the frequency $\omega^{\prime}$ of the wave in the electron frame is related to its frequency $\omega$ in the lab frame as follows:

$$
\omega^{\prime}=\gamma_{0} \omega\left(1-v_{0} / c \cos \theta\right) .
$$

This is the general relativistic Doppler-shift equation. For narrow angles $(\theta \ll 1)$, we can approximate it as

$$
\begin{aligned}
\omega^{\prime} & \approx \gamma_{0} \omega\left(1-\frac{v_{0}}{c}+\frac{v_{0}}{c} \frac{\theta^{2}}{2}\right) \\
& \approx \gamma_{0} \omega \frac{1-\left(\frac{v_{0}}{c}\right)^{2}+\left(1+\frac{v_{0}}{c}\right) \frac{v_{0}}{c} \frac{\theta^{2}}{2}}{1+\frac{v_{0}}{c}} \\
& \approx \omega \frac{1+\left(1+\frac{v_{0}}{c}\right) \frac{v_{0}}{c} \frac{\left(\gamma_{0} \theta\right)^{2}}{2}}{\gamma_{0}\left(1+\frac{v_{0}}{c}\right)} .
\end{aligned}
$$

Moreover, under the assumption of relativistic speed $\left(v_{0} / c \approx 1\right)$ and rearranging the terms, this equation further simplifies to

$$
\omega \approx \omega^{\prime} \frac{2 \gamma_{0}}{1+\left(\gamma_{0} \theta\right)^{2}}
$$


[1] The Science and Technology of Undulators and Wigglers (Oxford University Press, New York, 2004), Chap. 1.

[2] H. Motz, Applications of the radiation from fast electron beams, J. Appl. Phys. 22, 527 (1951).

[3] H. Motz, W. Thon, and R. N. Whitehurst, Experiments on radiation by fast electron beams, J. Appl. Phys. 24, 826 (1953).

[4] P. Emma, R. Akre1, J. Arthur, R. Bionta, C. Bostedt, and J. Bozek, First lasing and operation of an ångstromwavelength free-electron laser, Nat. Photonics 4, 641 (2010).

[5] T. Ishikawa, H. Aoyagi, T. Asaka, Y. Asano, N. Azumi, and T. Bizen, A compact X-ray free-electron laser emitting in the sub-ångström region, Nat. Photonics 6, 540 (2012).

[6] Synchrotron Radiation Instrumentation, edited by $\mathrm{T}$. Warwick, J. Arthur, H. A. Padmore, and J. Stöhr, AIP Conf. Proc. No. 705 (AIP, San Francisco, 2003).

[7] R. Pantell, G. Soncini, and H. Puthoff, Stimulated photonelectron scattering, IEEE J. Quantum Electron. 4, 905 (1968).

[8] S. Tantawi, M. Shumail, J. Neilson, G. Bowden, C. Chang, E. Hemsing, and M. Dunning, Experimental demonstration of a tunable microwave undulator, Phys. Rev. Lett. 112, 164802 (2014).

[9] Particle Accelerator Physics (Springer, New York, 2007), Chap. 20.
[10] The Science and Technology of Undulators and Wigglers (Oxford University Press, New York, 2004), Chap. 4.

[11] K. Batchelor, Microwave undulator, in Proceedings of the Linear Accelerator Conference, Stanford, California, 1986 (SLAC, Stanford, 1986), pp. 272-275.

[12] C. Pellegrini, X-Band microwave undulators for short wavelength free-electron lasers, AIP Conf. Proc. 807, 30 (2006).

[13] M. Shumail, G. Bowden, C. Chang, J. Neilson, and S. Tantawi, Application of the balanced hybrid mode in overmoded corrugated waveguides to short wavelength dynamic undulators, in Proceedings of the 2nd International Particle Accelerator Conference, San Sebastián, Spain (EPS-AG, Spain, 2011), pp. 3326-3328.

[14] Classical Electrodynamics (John Wiley \& Sons, Inc., New York, 1999), Chap. 11.

[15] Classical Electrodynamics (John Wiley \& Sons, Inc., New York, 1999), Chap. 12.

[16] Particle Accelerator Physics (Springer, New York, 2007), Chap. 21.

[17] Free-Electron Lasers in the Ultraviolet and X-ray Regime: Physical Principles, Experimental Results, Technical Realization (Springer, New York, 2014), Chap. 2.

[18] The Science and Technology of Undulators and Wigglers (Oxford University Press, New York, 2004), Chap. 3.

[19] Classical Electrodynamics (John Wiley \& Sons, Inc., New York, 1999), Chap. 14. 